\title{
Número de envoltória em classes de grafos orientados*
}

\author{
J. Araujo ${ }^{1}$, P. Arraes ${ }^{1}$ \\ ${ }^{1}$ ParGO - Departamento de Matemática - Universidade Federal do Ceará (UFC) \\ julio@mat.ufc.br, pedro.arraes@alu.ufc.br
}

\begin{abstract}
In this paper we study the hull number for some classes of oriented graphs. First we present an upper bound for the hull number of tournaments, and a tournament for which that bound is attained. Next we prove that the hull number problem is NP-complete for bipartite oriented graphs. For that we use the result of [Araujo et al. 2013], which asserts that the hull number problem is NP-complete for bipartite non-oriented graphs. After that we show a charecterization for the smallest hull set of a tree. Moreover, we generalize this result by showing a polynomial-time algorithm that computes the hull number of any oriented cactus graph.
\end{abstract}

Resumo. Neste trabalho estudamos o número de envoltória para algumas classes de grafos orientados. Primeiramente apresentamos um limitante superior para o número de envoltória restrito a torneios, além de um torneio para o qual atingimos esse limite. Em seguida provamos que esse problema é NPcompleto para grafos bipartidos orientados. Para tanto utilizamos o resultado de [Araujo et al. 2013], o qual afirma que tal problema é NP-completo para grafos bipartidos não-orientados. Depois mostramos uma caraterização para o menor conjunto de envoltória de uma árvore orientada. Além disso, generalizamos esse resultado ao mostrar um algoritmo de tempo polinomial para calcular o número de envoltória de qualquer grafo cacto orientado.

\section{Introdução}

Nessa seção apresentaremos algumas definições básicas de grafos orientados e o suficiente para se compreender o problema do número de envoltória. Para outras definições em grafos, veja [West 2000]; para definições sobre algoritmos e complexidade computacional, sugere-se [Garey and Johnson 1990].

Um grafo orientado é um grafo direcionado cujo grafo subjacente é simples. Dados um grafo orientado $D$ e $u, v \in V(D)$, um $(u, v)$-caminho direcionado $P$ é um subgrafo orientado de $D$ tal que $V(P)=\left\{u=v_{1}, v_{2}, \ldots, v_{k}=v\right\}$ e $A(P)=\left\{\left(v_{i}, v_{i+1}\right) \mid\right.$ $1 \leq i \leq k-1\}$; esse, por sua vez, é denotado por $\left(v_{1}, v_{2}, \ldots, v_{k}\right)$. Um ciclo direcionado $C$ é um ciclo orientado tal que $V(C)=\left\{v_{1}, v_{2}, \ldots, v_{k}\right\}$ e $A(C)=\left\{\left(v_{i}, v_{i+1}\right) \mid 1 \leq i \leq\right.$ $k-1\} \cup\left\{\left(v_{k}, v_{1}\right)\right\}$.

Dados um grafo orientado $D$ e vértices $u, v \in V(D)$, um $(u, v)$-geodésico é um caminho direcionado de $u$ para $v$ com menor comprimento possível, enquanto $d_{D}(u, v)$ denota a quantidade de $\operatorname{arcos}$ num $(u, v)$-geodésico. Perceba que um $(u, v)$-geodésico não é um $(v, u)$-geodésico (e vice-versa) e pode acontecer de $d_{D}(u, v) \neq d_{D}(v, u)$.

${ }^{*}$ Esta pesquisa foi financiada pelo CNPq sob projetos 459466/2014-3, 310234/2015-8 e 401519/2016-3. 
A função de intervalo $I: \mathcal{P}(V(D)) \rightarrow \mathcal{P}(V(D))$ aplicada em $S \subseteq V(D)$ retorna o conjunto $I[S]$, composto pelos vértices de todos os $(u, v)$-geodésicos e de todos os $(v, u)$-geodésicos com $u, v \in S$. Para todo inteiro $n \geq 2$ definimos $I^{n}[S]:=I\left[I^{n-1}[S]\right]$ e $I^{0}[S]=S$. Um conjunto $S$ é convexo se $I[S]=S$, ou seja, para todo vértice $w$ em algum $(u, v)$-geodésico com $u, v \in S$ temos $w \in S$. Em contrapartida, $S$ é dito co-convexo se $V(D) \backslash S$ é convexo. A envoltória de $S \subseteq V(D)$, denotada por $[S]$, é o menor conjunto convexo que contém $S$. Esse pode ser obtido aplicando a função de intervalo iterativamente em $S$ até obtermos um conjunto $S^{\prime}$ tal que $I\left[S^{\prime}\right]=S^{\prime}$. Tal conjunto sempre é atingido pois, como os grafos tratados aqui são finitos, em alguma iteração deixaremos de adicionar vértices. Isso quer dizer que existe um natural mínimo $n$ tal que $I^{n+1}[S]=I^{n}[S]=[S]$.

Quando a envoltória de um $S \subseteq V(D)$ é todo o conjunto de vértices do grafo orientado, dizemos que $S$ é um conjunto de envoltória de $D$. Além disso, se $S$ for um conjunto de envoltória de $D$ mínimo, definimos o número de envoltória de $D$ por $\overrightarrow{h n}(D):=|S|$. De forma similar, $S$ é dito um conjunto geodético de $D$ quando $I[S]=V(D)$ e, no caso em que esse é mínimo, definimos o número geodético de $D$ por $\overrightarrow{g n}(D):=|S|$.

Em grafos orientados, existem vértices que exercem um papel importante no problema do número de envoltória: os extremais. Esses podem ser de um dos três tipos a seguir: transmissor: $d_{D}^{-}(v)=0$ e $d_{D}^{+}(v)>0$; receptor: $d_{D}^{+}(v)=0$ e $d_{D}^{-}(v)>0$; e transitivo: $d_{D}^{-}(v)>0, d_{D}^{+}(v)>0$ e, para qualquer par de $\operatorname{arcos}(u, v),(v, w) \in A(D)$, temos $(u, w) \in A(D)$.

Lema 1 ([Chartrand et al. 2003]). Todo conjunto de envoltória (geodético) de um grafo orientado conexo $D$ deve conter os vértices extremais de $D$. Em particular, se o conjunto de vértices extremais for um conjunto de envoltória (geodético), então esse é o único conjunto de envoltória (geodético) mínimo.

A maioria dos trabalhos na literatura sobre Convexidade em Grafos lida com o caso não-orientado. Ademais, os que lidam com o caso orientado focam nas orientações de grafos cujo número de envoltória é ou o maior ou o menor possível.

Nossa primeira contribuição é um limitante superior para $\overrightarrow{h n}(D)$ quando $D$ é um torneio, além de um exemplo no qual esse é atingido. Em seguida mostramos que, restrito à classe de bipartidos orientados, o problema do número de envoltória é NP-completo.

Depois provamos que o conjunto de vértices extremais é um conjunto de envoltória mínimo para grafos árvore orientados. Para a classe de cactos orientados, mostramos como obter um conjunto de envoltória mínimo. Finalmente, discutimos os resultados obtidos e apresentamos problemas em aberto na última seção.

\section{Torneios}

Um torneio é um grafo completo orientado. Nossa abordagem inicial foi tomar a envoltória do conjunto composto pelos extremais e analisar os vértices que não fazem parte dela. Observamos que, para um subconjunto $\mathcal{S} \subset V(D)$ qualquer, os vértices extremais de $\mathcal{S}$ não influenciam na adição de outros vértices em $I[\mathcal{S}]$. Veja que, aplicando esse mesmo argumento mais $n-1$ vezes, podemos afirmar o mesmo para $I^{n}[\mathcal{S}]$. 
Portanto, sendo $S$ o conjunto composto pelos vértices extremais do torneio $D, \mathcal{S}$ é um conjunto de envoltória de $D$ se $S \subseteq \mathcal{S}$ e $[\mathcal{S} \backslash S]_{D}=V(D) \backslash S$. Ou seja, nosso problema se resume a encontrar um conjunto de vértices (não extremais) cuja envoltória seja composta por todos os vértices não extremais. Abaixo mostramos que, para obter todos os vértices de $V(D)$, além dos extremais precisamos de no máximo dois terços dos outros vértices.

Proposição 1. Seja $D$ um torneio e $S$ o conjunto de vértices extremais de $D$. Então $\overrightarrow{h n}(D) \leq|S|+\frac{2}{3}(n(D)-|S|)$ e esse limite é apertado.

Para obter um torneio no qual o limite acima é atingido basta fazer o produto lexicográfico de $K_{3} \equiv C_{3}$, orientado de forma a ser um ciclo direcionado, com um $K_{5}$, orientado de forma transitiva, de modo que cada vértice do segundo é substituido pelo $C_{3}$ direcionado.

\section{Bipartidos}

O objetivo dessa seção é mostrar que, dados um grafo orientado $D$ e um inteiro positivo $k$, decidir se $\overrightarrow{h n}(G) \leq k$ é um problema NP-completo, mesmo que $G$ seja bipartido. Para tanto, fazemos uso do resultado abaixo, o qual mostra a dificuldade desse problema para grafos bipartidos.

Teorema 1 ([Araujo et al. 2013]). Dados um grafo $G$ e um inteiro positivo $k$, decidir se $\mathrm{hn}(G) \leq k$ é um problema NP-completo, mesmo se $G$ for um grafo bipartido.

O método que usaremos para provar o resultado final é o seguinte. Dado um grafo bipartido $G$ podemos construir um bipartido orientado $G_{\overrightarrow{C 4}}$, pela substituição de cada aresta por um ciclo direcionado com 4 vértices, cujo número de envoltória é o mesmo.

Teorema 2. Se $G$ for um grafo bipartido, então $h n(G)=\overrightarrow{h n}\left(G_{\overrightarrow{C 4}}\right)$.

Corolário 1. Dados um grafo bipartido orientado $G$ e um inteiro positivo $k$, decidir se $\overrightarrow{h n}(G) \leq k$ é um problema NP-completo.

\section{4. Árvores e Cactos}

Antes de mostrarmos um algoritmo polinomial para determinar o número de envoltória de um cacto orientado, vamos mostrar um resultado sobre uma subclasse de cactos: as árvores. Para essa classe, tentamos a mesma abordagem dos torneios: tomar a envoltória dos extremais.

Proposição 2. Seja $D$ uma árvore orientada e $S \subseteq V(D)$ o conjunto formado pelos vértices extremais de $D$. $S$ é um conjunto de envoltória e geodético de $D$.

Um grafo $G$ é dito um cacto se cada um de seus blocos ou é um ciclo induzido ou é uma aresta. Uma maneira mais informal de definir essa classe é que quaisquer dois subgrafos ciclos do grafo possuem no máximo um vértice em comum, o qual seria uma articulação. Descobrimos que além dos extremais existem ciclos no cacto tais que todo conjunto de envoltória do grafo deve ter pelo menos um de seus vértices. A esses ciclos damos o nome de insatisfeitos. Além disso, vimos que esses vértices não são apenas necessários num conjunto de envoltória, eles são suficientes.

Teorema 3. Seja $D$ um cacto orientado. Então, existe um conjunto de envoltória mínimo $S$ de $D$ composto pelos vértices extremais e exatamente um vértice (não-extremal) de 
cada ciclo insatisfeito tal que $I^{2}[S]=V(D)$ e todos os vértices que podem não estar em $I[S]$ pertencem a ciclos satisfeitos. Consequentemente, todo conjunto de envoltória mínimo $S$ de $D$ é composto pelos vértices extremais e exatamente um vértice (não-extremal) de cada ciclo insatisfeito.

\section{Conclusões}

Nas Seções 2 e 4, abordamos o problema do número de envoltória da mesma forma: analisando a envoltória dos extremais e formulando uma maneira de obter os demais. Um caminho natural é tentar utilizar esse método para encontrar mais resultados desse parâmetro em outras classes de grafos orientados.

Seguindo essa linha de raciocínio, será possível aplicar um método similar utilizado na Seção 3 em subclasses de bipartidos para provar a NP-completude? Por exemplo, foi demonstrado em [Albenque and Knauer 2016] que encontrar o número de envoltória também é NP-difícil para cubos parciais. Cubos parciais são subgrafos isométricos de hipercubos, os quais por sua vez são bipartidos.

Além disso, ainda do ponto de vista de Complexidade Computacional, há inúmeras classes de grafos orientados a serem abordadas, por exemplo: cordais, planares, grafos com largura em árvore limitada, etc. Também não conhecemos resultados do ponto de vista de Complexidade Parametrizada para problemas de convexidade em grafos orientados. Finalmente, só encontramos na literatura resultados sobre os parâmetros número de envoltória e número geodético, ou seja, todos os demais parâmetros de convexidade ainda devem ser estudados no caso orientado [Chartrand et al. 2002, Duchet 1988].

\section{Referências}

Albenque, M. and Knauer, K. (2016). Convexity in partial cubes: The hull number. Discrete Mathematics, 339(2):866 - 876.

Araujo, J., Campos, V., Giroire, F., Nisse, N., Sampaio, L., and Soares, R. (2013). On the hull number of some graph classes. Theoretical Computer Science, 475(Supplement C): $1-12$.

Chartrand, G., Fink, J. F., and Zhang, P. (2003). The hull number of an oriented graph. International Journal of Mathematics and Mathematical Science, 2003(36):2265-2275.

Chartrand, G., Wall, C., and Zhang, P. (2002). The convexity number of a graph. Graphs and Combinatorics, 18(2):209-217.

Duchet, P. (1988). Convex sets in graphs, ii. minimal path convexity. Journal of Combinatorial Theory, Series B, 44(3):307-316.

Garey, M. R. and Johnson, D. S. (1990). Computers and Intractability; A Guide to the Theory of NP-Completeness. W. H. Freeman \& Co., New York, NY, USA.

West, D. B. (2000). Introduction to Graph Theory. Prentice Hall, 2 edition. 